\title{
Methylation patterns in serum DNA for early identification of disseminated breast cancer
}

Martin Widschwendter ${ }^{1 *}$ (D, Iona Evans ${ }^{1}$, Allison Jones ${ }^{1}$, Shohreh Ghazali ${ }^{1}$, Daniel Reisel ${ }^{1}$, Andy Ryan ${ }^{1}$, Aleksandra Gentry-Maharaj ${ }^{1}$, Michal Zikan ${ }^{2}$, David Cibula ${ }^{2}$, Johannes Eichner ${ }^{3}$, Marianna Alunni-Fabbroni ${ }^{4}$, Julian Koch ${ }^{4}$, Wolfgang J. Janni ${ }^{5}$, Tobias Paprotka ${ }^{6}$, Timo Wittenberger ${ }^{3}$, Usha Menon ${ }^{1}$, Benjamin Wahl ${ }^{6,7 \dagger}$, Brigitte Rack ${ }^{4,5+}$ and Harri Lempiäinen ${ }^{3+}$

\begin{abstract}
Background: Monitoring treatment and early detection of fatal breast cancer (BC) remains a major unmet need. Aberrant circulating DNA methylation (DNAme) patterns are likely to provide a highly specific cancer signal. We hypothesized that cell-free DNAme markers could indicate disseminated breast cancer, even in the presence of substantial quantities of background DNA.

Methods: We used reduced representation bisulfite sequencing (RRBS) of 31 tissues and established serum assays based on ultra-high coverage bisulfite sequencing in two independent prospective serum sets $(n=110)$. The clinical use of one specific region, EFC\#93, was validated in 419 patients (in both pre- and post-adjuvant chemotherapy samples) from SUCCESS (Simultaneous Study of Gemcitabine-Docetaxel Combination adjuvant treatment, as well as Extended Bisphosphonate and Surveillance-Trial) and 925 women (pre-diagnosis) from the UKCTOCS (UK Collaborative Trial of Ovarian Cancer Screening) population cohort, with overall survival and occurrence of incident breast cancer (which will or will not lead to death), respectively, as primary endpoints.

Results: A total of $18 \mathrm{BC}$ specific DNAme patterns were discovered in tissue, of which the top six were further tested in serum. The best candidate, EFC\#93, was validated for clinical use. EFC\#93 was an independent poor prognostic marker in pre-chemotherapy samples (hazard ratio [HR] for death $=7.689$ ) and superior to circulating tumor cells $(\mathrm{CTCS})$ (HR for death =5.681). More than 70\% of patients with both CTCS and EFC\#93 serum DNAme positivity in their pre-chemotherapy samples relapsed within five years. EFC\#93-positive disseminated disease in post-chemotherapy samples seems to respond to anti-hormonal treatment. The presence of EFC\#93 serum DNAme identified $42.9 \%$ and 25\% of women who were diagnosed with a fatal BC within 3-6 and 6-12 months of sample donation, respectively, with a specificity of $88 \%$. The sensitivity with respect to detecting fatal BC was $\sim 4$-fold higher compared to non-fatal BC.

Conclusions: Detection of EFC\#93 serum DNAme patterns offers a new tool for early diagnosis and management of disseminated breast cancers. Clinical trials are required to assess whether EFC\#93-positive women in the absence of radiological detectable breast cancers will benefit from anti-hormonal treatment before the breast lesions become clinically apparent.
\end{abstract}

Keywords: Cell-free DNA, DNA methylation, Serum DNA, Breast cancer, Early diagnosis, Personalized treatment

\footnotetext{
* Correspondence: M.Widschwendter@ucl.ac.uk

${ }^{\dagger}$ Equal contributors

'Department of Women's Cancer, UCL Elizabeth Garrett Anderson Institute

for Women's Health, University College London, Medical School Building,

74 Huntley Street, London WC1E 6AU, UK

Full list of author information is available at the end of the article
} 


\section{Background}

Breast cancer $(\mathrm{BC})$ is by far the most frequently occurring cancer in women. Every year 522,000 women die from BC [1].

Mammography is used as a screening tool for early diagnosis but has its limitations due to over-diagnosis and a modest impact on mortality [2]. Recent evidence demonstrates that dissemination might occur during the very early stages of tumor evolution and before clinical manifestation of the cancer in the breast [3]. The analyses of circulating markers in order to identify women with disseminated disease before diagnosis have not been successful [4].

Numerous studies have demonstrated that patients with disseminated tumor cells in the bone marrow [5-7] or circulating tumor cells (CTCs) [8-12] have an inferior prognosis. The immunocytochemical detection of CTCs is reliant upon the isolation of intact cells.

Adjuvant systemic treatment has reduced BC mortality over the last two to three decades [13]. The current strategy guiding administration of adjuvant systemic treatment is reliant upon primary tumor characteristics. However, systemic relapse and subsequent death are caused by disseminated disease whose biological properties may be very different to those comprising the primary tumor [14].

Recently, markers based on DNA shed from tumor cells have shown great promise in monitoring treatment response and predicting prognosis [15-19]. However, efforts to characterize the cancer genome have shown that only a few genes are frequently mutated in cancer and the site of mutation per gene differs across tumors [20]. A further limitation is that current technology only allows for the detection of a mutant allele fraction of $0.1 \%[15,21]$.

Over the last decade, DNA methylation (DNAme) has been shown to be a hallmark of cancer [22] and occurs very early in BC development [23]. DNAme is centered around specific regions (CpG islands) [22] and is chemically and biologically stable. This enables the development of early detection tools and personalized treatment, based upon the analysis of cell-free DNA contained within serum or plasma [24-29]. However, two major challenges have to be overcome: (1) the very low abundance of cancer-DNA in the blood; and (2) the high level of "background DNA" shed from white blood cells (WBC) [30] in banked samples.

To date, virtually all research work has been carried out in relatively small studies and focused on the analyses of cell-free DNAme in metastatic/relapsed breast cancers using markers from previously published studies [31]. In our study we: (1) used an epigenomewide approach to identify new markers which indicate disseminated breast cancer; (2) analyzed the top marker in 419 primary non-metastatic patients before (i.e. immediately after resection of the primary breast cancer) and after adjuvant chemotherapy; and, most importantly (3) analyzed the marker in 925 healthy women who either remained healthy or developed fatal or non-fatal $\mathrm{BC}$ within the first three years after serum sample donation.

\section{Methods}

\section{Patients and sample collection}

We used a total of 31 tissues and 1869 serum samples (Fig. 1). In Phase 1, we analyzed breast cancer tissue and WBCs in order to identify breast cancer specific DNAme markers. In Phase 2, we established serum DNAme assays using serum sets 1 and 2, collected from women attending hospitals in London, Munich, and Prague where they were invited and consented. Blood samples (20-40 mL) were obtained (in VACUETTE ${ }^{\oplus}$ Z Serum Sep Clot Activator tubes), centrifuged at $3000 \mathrm{rpm}$ for $10 \mathrm{~min}$, and serum collected and stored at $-80{ }^{\circ} \mathrm{C}$. Finally, Phase 3 was initiated to validate the top marker performance by using serum samples from two large clinical studies: (1) from 419 patients recruited within the SUCCESS trial [10] (ClinicalTrial.gov registration ID is NCT02181101), where bloods were taken before and after chemotherapy and (within $96 \mathrm{~h}$ ) sent to the laboratory for CTC assessment and serum samples stored (Additional file 1: Figure S1); and (2) from UKCTOCS [32] (ClinicalTrial.gov registration ID is NCT00058032), where serum samples were used from: (i) 229 women diagnosed with $\mathrm{BC}$ within the first three years after serum sample donation and subsequently died during follow-up; (ii) 231 matched women who developed $\mathrm{BC}$ within three years after sample donation and were alive at the end of follow-up; and (iii) 465 women who did not develop $\mathrm{BC}$ within five years after sample donation (Additional file 1: Figure S2). Blood samples from all UKCTOCS volunteers were spun down for serum separation after having been transported at room temperature from trial centers to the central laboratory. The median time between sample collection and centrifugation was $22.1 \mathrm{~h}$. Only $1 \mathrm{~mL}$ of serum per UKCTOCS volunteer was available. All patients provided written informed consent.

\section{Isolation and bisulfite modification of DNA}

DNA was isolated from tissue and serum samples at GATC Biotech (Konstanz, Germany). Tissue DNA was quantified using NanoDrop ${ }^{\mathrm{TM}}$ and Qubit $^{\mathrm{TM}}$, and the size was assessed by agarose gel electrophoresis. Serum DNA was quantified using the Agilent Fragment Analyzer and the High Sensitivity Large Fragment Analysis Kit (AATI, USA). DNA was bisulfite converted at GATC Biotech. 


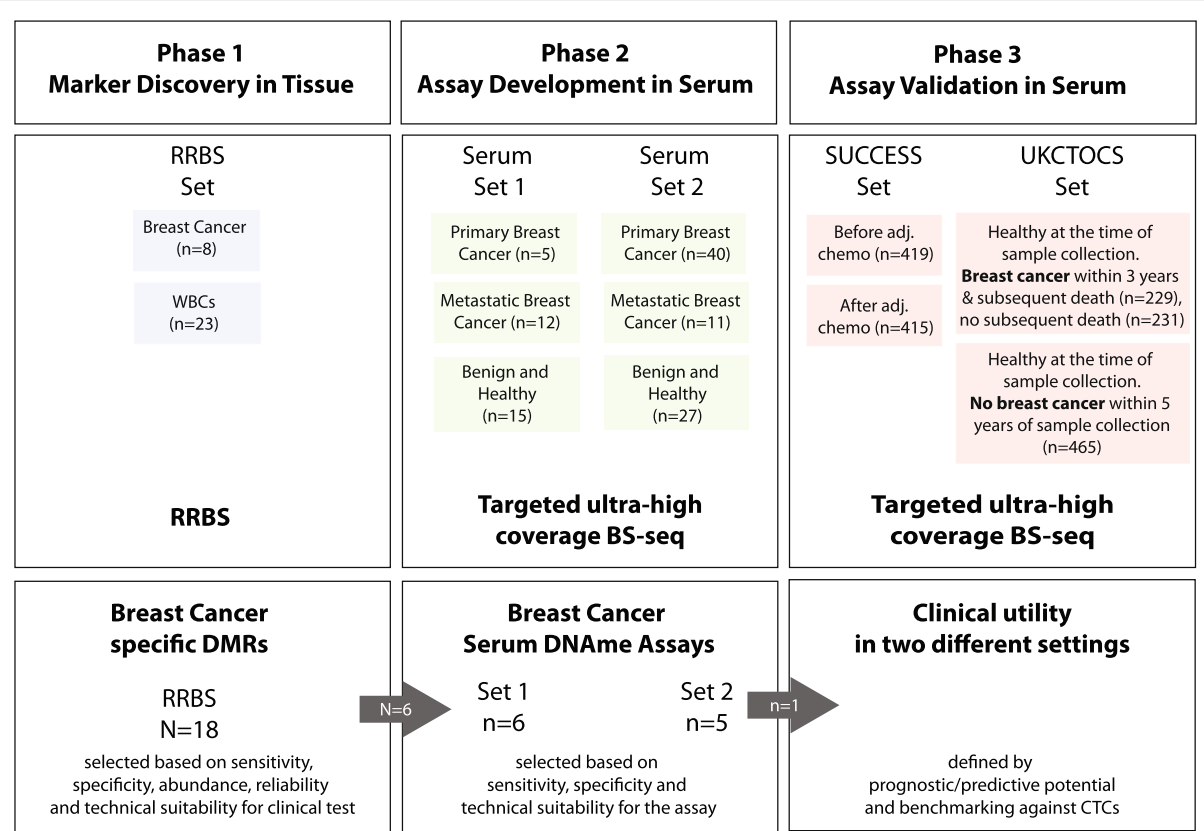

Fig. 1 Study design. Using reduced representation bisulfite sequencing (RRBS), 31 human tissue samples were analyzed to identify a total of 18 regions which underwent thorough technical validation. Six regions were selected whose methylation status has been analyzed in two sets consisting of 110 serum samples. One marker (EFC\#93) has been validated in two independent settings: (1) in SUCCESS study serum samples from BC patients before and after chemotherapy; and (2) in UKCTOCS serum samples from women before BC diagnosis (within three years) or who remained healthy for five years

\section{DNAme analysis in tissue}

Genome-wide methylation analysis was performed by reduced representation bisulfite sequencing (RRBS) at GATC Biotech. DNA was digested with MspI followed by size selection of the library, providing enhanced coverage for the CpG-rich regions [33, 34]. The digested DNA was adapter-ligated, bisulfite-modified, and polymerase chain reaction (PCR)-amplified. The libraries were sequenced on Illumina's HiSeq 2500. Analysis of the first samples sequenced with a 100-bp paired-end mode showed that the library insert size was small. Therefore, the remaining samples were sequenced with a 50-bp paired-end mode. Using Genedata Expressionist ${ }^{\oplus}$ for Genomic Profiling v9.1, we established a bioinformatics pipeline for the detection of cancer specific differentially methylated regions (DMRs). The most promising DMRs were taken forward for the development and validation of serum-based clinical assays.

\section{Targeted ultra-high coverage bisulfite sequencing of serum DNA}

Targeted bisulfite sequencing libraries were prepared at GATC Biotech. Bisulfite modification was performed with $1 \mathrm{~mL}$ serum equivalent. A two-step PCR approach was used to test up to three different markers per modified DNA sample. The first PCR amplifies the target region and adds linker sequences which are used in the second PCR to add barcodes for multiplexing and sequences needed for sequencing. Ultra-high coverage sequencing was performed on Illumina's MiSeq or HiSeq 2500 with a 75-bp or 125-bp paired-end mode, respectively.

\section{Data analyses}

Genedata Expressionist ${ }^{\circledR}$ for Genomic Profiling was used to map reads to human genome version hg19, identify regions with tumor-specific methylation patterns, quantify the occurrence of those patterns, and calculate relative pattern frequencies per sample. Pattern frequencies were calculated as number of reads containing the pattern divided by total reads covering the pattern region. Methylation patterns are represented in terms of a binary string, where the methylation state of each CpG site is denoted by "1" if methylated or " 0 " if unmethylated. The algorithm that we developed scans the whole genome and identifies regions that contain at least ten aligned paired-end reads. These read bundles are split into smaller regions of interest (ROIs) which contain at least $4 \mathrm{CpGs}$ in a stretch of $<150 \mathrm{bp}$. For each region and tissue/sample, the absolute frequency (number of supporting reads) for all observed methylation patterns was determined (Fig. 2a). This led to the discovery of tens of millions of patterns per tissue/sample. The patterns were filtered in a multi-step procedure to identify the methylation patterns specifically occurring in tumor samples. To increase the sensitivity and specificity of our pattern discovery procedure, we pooled reads from different tumor or WBC samples and scored patterns based on over- 

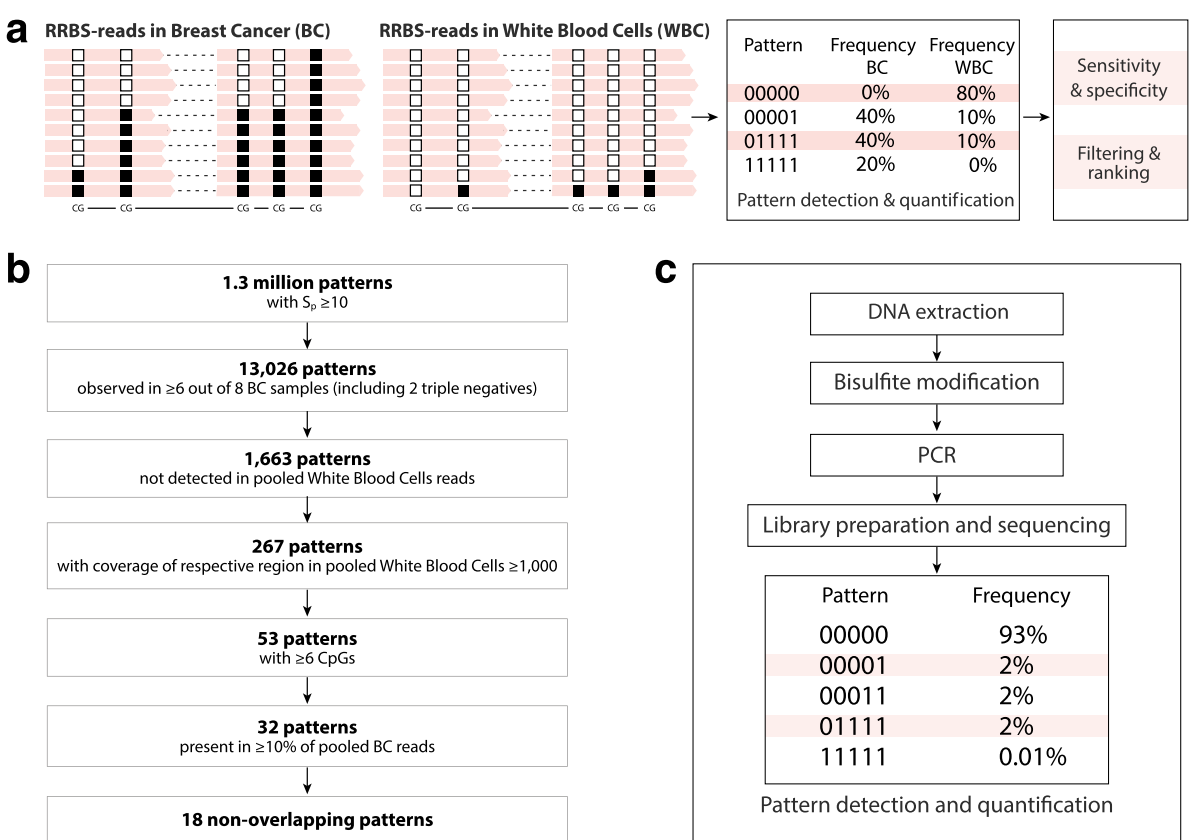

Fig. 2 Principles of methylation pattern discovery in tissue $(\mathbf{a}, \mathbf{b})$ and analyses in serum (c). a RRBS was used in tissue samples in order to identify CpG methylation patterns that are able to discriminate breast cancer from white blood cells (which were deemed to be the most abundant source of cell-free DNA). "0" represents an unmethylated CpG and "1" represents a methylated CpG. An example of region EFC\#93 is provided which is a 136-bp-long region containing five linked CpGs. The cancer pattern consists of reads in which all linked CpGs are methylated, indicated by "11111." b RRBS data have been processed through a bioinformatic pipeline to identify the most promising markers. c The principles of the serum DNA methylation assay

representation within tumor tissue. The results were summarized in a specificity score, $\mathrm{Sp}$, which reflects the cancer specificity of the patterns. After applying a cut-off of $\mathrm{Sp} \geq 10,1.3$ million patterns for $\mathrm{BC}$ remained and were further filtered according to the various criteria detailed in Fig. $2 \mathrm{~b}$ (further details are provided in Additional file 2).

The 95\% confidence intervals (CI) for sensitivity and specificity have been calculated according to the efficient-score method [35]. The endpoints were defined according to the STEEP criteria, with relapse-free survival and overall survival as the primary endpoints. The product-limit method according to Kaplan-Meier was used to estimate survival. The survival estimates in different groups were compared using the log-rank test. The Cox proportional hazards regression model was used for the analyses taking into account all variables simultaneously.

Further details on samples and methods can be found in Additional file 2.

\section{Results}

The samples, techniques, and purpose of the three phases used in this study (marker discovery, assay development, and assay validation) are summarized in Fig. 1. We first identified DMRs based on their methylation patterns and frequencies in relevant genomic regions, within a $\mathrm{BC}$ tissue panel. Methylation patterns with high specificity for breast cancer tissue were identified using the procedure described in Fig. $2 \mathrm{~b}$.

The selected $18 \mathrm{BC}$ specific patterns identified by RRBS, were further validated using bisulfite sequencing. Thirty-one bisulfite sequencing primer pairs (1-3 per region) were designed and technically validated to determine PCR efficiency and sensitivity. A dilution series obtained by mixing fully unmethylated (i.e. whole genome amplified DNA) with fully methylated DNA (i.e. whole genome amplified DNA treated with CpG methyltransferase) was used to select six reactions which showed good coverage after sequencing ( $>10^{4}$ reads) and sensitivity in highly diluted $\left(<1: 10^{4}\right)$ samples (Additional file 3: Table S1). The best six reactions were taken into Phase 2, for further testing and assay development, in prospectively collected serum sets. We used ultra-deep bisulfite sequencing to develop assays for these candidate regions in 32 serum samples from Serum Set 1 (Figs. 1 and 2c). Five of the six reactions showed good sensitivity and specificity (particularly when discriminating between metastatic and primary $\mathrm{BC})$, based on the abundance of tumor-specific patterns (see Additional file 1: Figure S3 for a complete overview of pattern counts from region EFC\#93) and were selected for further validation in Serum Set $2(n=78)$. 
DNA methylation marker EFC\#93, which was identified in RRBS as a region of ten linked CpGs methylated in $\mathrm{BC}$, was optimized to a pattern of five linked CpGs and showed the best sensitivity and specificity independently in Set 1 and 2 (Additional file 1: Figure S4). A statistically higher pattern frequency, for the optimized marker EFC\#93, was observed in the metastatic BC groups compared to the healthy/benign lesions or primary $\mathrm{BC}$ groups, in both Sets 1 and 2. This translates to an area under the curve (AUC) of a receiver operating characteristics (ROC) curve of $0.850(95 \% \mathrm{CI}=0.745-0.955, P=0.000004)$ and 0.845 (95\% CI $=0.739-0.952, P=0.000004)$ to discriminate healthy/benign lesions or primary $\mathrm{BC}$ from metastatic
$\mathrm{BC}$ in Set 1 and Set 2, respectively. When Set 1 and 2 data were combined, the pattern frequency threshold was set to 0.0008 (i.e. 8 in 10,000 reads demonstrated methylation at all CpGs in the EFC\#93 region), which led to a sensitivity of $60.9 \%$ and a specificity of $92.0 \%$ with respect to identifying metastatic BC (Additional file 1: Figure S4).

EFC\#93 was then validated for use as a prognostic and predictive $\mathrm{BC}$ marker in clinical trial samples (Fig. 1). As expected, due to delayed sample processing within these trials, serum samples from both SUCCESS and UKCTOCS contained high levels of contaminating WBC DNA, leading to dilution of the cancer signal (Additional file 1: Figure S5). In order to adjust for this, we made an a priori decision to

Table 1 SUCCESS patient characteristics before and after chemotherapy for EFC\#93 serum DNAme

\begin{tabular}{|c|c|c|c|c|c|c|c|}
\hline \multirow[t]{2}{*}{ Characteristic } & & \multicolumn{3}{|c|}{ Before chemotherapy } & \multicolumn{3}{|c|}{ After chemotherapy } \\
\hline & & EFC\#93- (\%) & EFC\#93+ (\%) & $P$ value $^{a}$ & EFC\#93- (\%) & EFC\#93+ (\%) & $P$ value \\
\hline Patients ( $\mathrm{n}$ ) & & $385(91.9)$ & $34(8.1)$ & & $371(89.4)$ & $44(10.6)$ & \\
\hline Age $($ mean $\pm S D)$ & & $53.7 \pm 10.3$ & $55.2 \pm 10.1$ & 0.380 & $53.5 \pm 10.4$ & $56.2 \pm 9.3$ & 0.097 \\
\hline \multirow[t]{2}{*}{ Menopausal status } & Premenopausal & $165(42.9)$ & $15(44.1)$ & 1.000 & $165(44.5)$ & $15(34.1)$ & 0.202 \\
\hline & Postmenopausal & $220(57.1)$ & $19(55.9)$ & & $206(55.5)$ & $29(65.9)$ & \\
\hline \multirow[t]{2}{*}{ Stage $(T)$} & $\mathrm{T} 1$ & $158(41.0)$ & $9(26.5)$ & 0.110 & $157(42.3)$ & $10(22.7)$ & 0.014 \\
\hline & $\mathrm{T} 2-4$ & $227(59.0)$ & $25(73.5)$ & & $214(57.7)$ & $34(77.3)$ & \\
\hline \multirow[t]{2}{*}{ Nodes (N) } & NO & $130(33.9)$ & $7(20.6)$ & 0.130 & $124(33.4)$ & $13(30.2)$ & 0.735 \\
\hline & N1-3 & $254(66.1)$ & $27(79.4)$ & & $247(66.6)$ & $30(69.8)$ & \\
\hline \multirow[t]{2}{*}{ Histology } & Invasive ductal & $310(80.5)$ & $25(73.5)$ & 0.370 & $296(79.8)$ & $36(81.8)$ & 0.844 \\
\hline & Others & $75(19.5)$ & $9(26.5)$ & & $75(20.2)$ & $8(18.2)$ & \\
\hline \multirow[t]{2}{*}{ Grading } & Grade 1/2 & $199(51.7)$ & $16(47.1)$ & 0.721 & $190(51.2)$ & $23(52.3)$ & 1.000 \\
\hline & Grade 3 & $186(48.3)$ & $18(52.9)$ & & $181(48.8)$ & $21(47.7)$ & \\
\hline \multirow[t]{2}{*}{ Estrogen (ER) receptor } & ER- & $128(33.2)$ & $10(29.4)$ & 0.708 & $128(34.5)$ & $10(22.7)$ & 0.130 \\
\hline & ER+ & $257(66.8)$ & $24(70.6)$ & & $243(65.5)$ & $34(77.3)$ & \\
\hline \multirow[t]{2}{*}{ Progesterone (PR) receptor } & PR- & $155(40.4)$ & $11(32.4)$ & 0.465 & $150(40,5)$ & $16(36.4)$ & 0.629 \\
\hline & PR+ & $229(59.6)$ & $23(67.6)$ & & $220(59.5)$ & $28(63.6)$ & \\
\hline \multirow[t]{2}{*}{ HER2 status } & HER2- & $294(77.0)$ & $24(70.6)$ & 0.403 & $276(75.0)$ & $38(86.4)$ & 0.132 \\
\hline & HER2+ & $88(23.0)$ & $10(29.4)$ & & $92(25.0)$ & $6(13.6)$ & \\
\hline \multirow[t]{2}{*}{ Surgery } & Breast conserving & $273(70.9)$ & $16(47.1)$ & 0.006 & $264(71.2)$ & $23(52.3)$ & 0.015 \\
\hline & Mastectomy & $112(29.1)$ & $18(52.9)$ & & $107(28.8)$ & $21(47.7)$ & \\
\hline \multirow[t]{2}{*}{ Chemotherapy } & FEC-D & $193(50.1)$ & $18(52.9)$ & 0.858 & $186(50.1)$ & $22(50.0)$ & 1.000 \\
\hline & FEC-DG & $192(49.9)$ & $16(47.1)$ & & $185(49.9)$ & $22(50.0)$ & \\
\hline \multirow[t]{2}{*}{ Bisphosphonates } & Zometa 2 years & $193(50.1)$ & $17(50.0)$ & 1.000 & $185(49.9)$ & $23(52.3)$ & 0.874 \\
\hline & Zometa 5 years & $192(49.9)$ & $17(50.0)$ & & $186(50.1)$ & $21(47.7)$ & \\
\hline \multirow[t]{4}{*}{ Circulating tumor cells (CTCs) } & CTC- before chemo & $316(82.1)$ & $20(58.8)$ & 0.003 & $303(81.7)$ & $32(72.3)$ & 0.160 \\
\hline & CTC+ before chemo & $69(17.9)$ & $14(41.2)$ & & $68(18.3)$ & $12(27.7)$ & \\
\hline & CTC- after chemo & $304(79.0)$ & $27(79.4)$ & 1.000 & $302(81.4)$ & $28(63.6)$ & 0.009 \\
\hline & CTC+ after chemo & $81(21.0)$ & $7(20.6)$ & & 69 (18.6) & 16 (36.4) & \\
\hline
\end{tabular}

EFC\#93 serum DNAme was deemed positive (+ve) at or above a pattern frequency of 0.00008

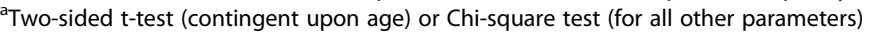

Information on N, PR and HER2 is missing from 1, 1 and 3 patients, respectively. Serum DNAme was not analysed for 4 post-treatment samples

FEC-D fluorouracil-epirubicin-cyclophosphamide $\left(500 / 100 / 500 \mathrm{mg} / \mathrm{m}^{2}\right.$, FEC) followed by docetaxel $\left(100 \mathrm{mg} / \mathrm{mg}^{2}\right)$, FEC-DG fluorouracil-epirubicin-cyclophosphamide $\left(500 / 100 / 500 \mathrm{mg} / \mathrm{m}^{2}, \mathrm{FEC}\right)$ followed by gemcitabine $\left(1000 \mathrm{mg} / \mathrm{m}^{2} \mathrm{~d} 1,8\right)$-docetaxel $\left(75 \mathrm{mg} / \mathrm{m}^{2}\right), S D$ standard deviation 
reduce the threshold for EFC\#93 pattern frequency by a factor of 10 to 0.00008 (i.e. $8 / 100,000$ reads demonstrated methylation at all five linked CpGs within the EFC\#93 region). Table 1 shows SUCCESS patient characteristics, correlated with EFC\#93 positivity/negativity, before and after chemotherapy. Using our predetermined threshold, EFC\#93 positivity was significantly associated with CTC presence, both before and after chemotherapy (Chi-square test, $P<0.01$, Table 1) although ECF\#93 pattern frequencies were not significantly different in samples from patients with either no, 1-4, or $>4$ CTCs detected, respectively (Additional file 1: Figure S6). Patients who underwent breast-conserving therapy were more likely to be EFC\#93negative compared to patients who underwent a mastectomy; this is in all probability explained by the fact that patients which presented with larger tumors tended to be EFC\#93-positive and would not have been eligible for breast-conserving surgery. This is consistent with the findings that EFC\#93 positivity after chemotherapy is significantly $(P=0.014)$ less frequently observed in early stage (T1) compared to late stage (T2-4) cancers. None of the other clinical-pathological features correlated with cellfree DNA methylation of EFC\#93 (Table 1). EFC\#93 serum positivity before chemotherapy was a very strong marker of poor prognosis, for both relapse-free and overall survival
(Table 2 and Fig. 3a and b). This was independent of the prognostic capability of CTCs (Additional file 1: Figures S7 and S8). Hazard ratios (HRs) (95\% CI) for overall survival in the multivariable model were $5.973(2.634-13.542)$ and 3.623 (1.681-7.812) for EFC\#93 and CTCs, respectively (Table 2). Patients who were CTC-positive and EFC\#93positive had an extremely poor outcome, with $>70 \%$ of these patients relapsing within five years (Fig. $3 \mathrm{c}$ and d). Neither serum marker EFC\#93 nor CTCs alone were predictive of the outcome in samples collected after chemotherapy (Additional file 1: Figures S9 and S10).

To assess whether EFC\#93 serum DNAme can diagnose women with poor prognostic BC earlier, we analyzed serum samples from 925 women from our UKCTOCS cohort. The amount of DNA as well as the fragment length was dramatically higher than expected and correlated with the average UK temperature (Additional file 1: Figures S11 and S12); there was also a good correlation between DNA amount and fragment length (Additional file 1: Figure S13) indicating a substantial leak of blood cell DNA into the serum during the blood transport. Within this nested case/control setting, the women with BC (cases) had provided serum samples up to three years before diagnosis. Again, we a priori hypothesized that the high background levels of DNA

Table 2 Univariate and multivariable proportional hazards model for relapse-free and overall survival for SUCCESS serum samples

\begin{tabular}{|c|c|c|c|c|}
\hline \multirow[t]{3}{*}{ Characteristic } & \multicolumn{4}{|c|}{ Univariate analyses } \\
\hline & \multicolumn{2}{|l|}{ Relapse-free survival } & \multicolumn{2}{|l|}{ Overall survival } \\
\hline & HR $(95 \% \mathrm{Cl})$ & $P$ value & $\mathrm{HR}(95 \% \mathrm{Cl})$ & $P$ value \\
\hline Menopausal status, post vs pre & $1.323(0.750-2.333)$ & 0.335 & $2.872(1.164-7.086)$ & 0.022 \\
\hline Tumor size, T2-T4 vs T1 & $2.268(1.187-4.332)$ & 0.013 & $3.881(1.343-11.218)$ & 0.012 \\
\hline Lymph node involvement, N1-3 vs N0 & $1.645(0.861-3.142)$ & 0.132 & $3.012(1.045-8.683)$ & 0.041 \\
\hline Estrogen receptor (ER) status, ER- vs ER+ & $1.316(0.999-1.734)$ & 0.051 & $1.333(0.918-1.934)$ & 0.131 \\
\hline Progesterone receptor (PR) status, PR- vs PR+ & $1.180(0.897-1.554)$ & 0.237 & $1.219(0.839-1.772)$ & 0.298 \\
\hline HER2 status, HER2+ vs HER2- & $1.907(0.858-4.241)$ & 0.113 & $1.789(0.618-5.178)$ & 0.283 \\
\hline Grading, G3 vs G1/2 & $1.079(0.623-1.868)$ & 0.786 & $1.129(0.535-2.384)$ & 0.75 \\
\hline CTCs before chemo, CTC+ vs CTC- & $3.666(2.110-6.368)$ & $<0.0001$ & $5.681(2.686-12.014)$ & $<0.0001$ \\
\hline CTCs after chemo, CTC+ vs CTC- & $1.401(0.757-2.592)$ & 0.283 & $1.467(0.646-3.331)$ & 0.36 \\
\hline EFC\#93 before chemo, EFC\#93+ vs EFC\#93- & $4.912(2.613-9.233)$ & $<0.0001$ & $7.689(3.518-16.804)$ & $<0.0001$ \\
\hline \multirow[t]{4}{*}{ EFC\#93 after chemo, EFC\#93+ vs EFC\#93- } & $1.913(0.927-3.949)$ & 0.079 & $1.807(0.673-4.853)$ & 0.24 \\
\hline & \multicolumn{4}{|c|}{ Multivariable analyses } \\
\hline & \multicolumn{2}{|l|}{ Relapse-free survival } & \multicolumn{2}{|l|}{ Overall survival } \\
\hline & $\mathrm{HR}(95 \% \mathrm{Cl})$ & $P$ value & HR $(95 \% \mathrm{Cl})$ & $P$ value \\
\hline Menopausal status & $1.294(0.728-2.302)$ & 0.379 & $2.688(1.070-6.750)$ & 0.035 \\
\hline Tumor size & $1.763(0.914-3.401)$ & 0.091 & $2.945(1.009-8.597)$ & 0.048 \\
\hline Lymph node involvement & $1.442(0.750-2.775)$ & 0.273 & $2.242(0.765-6.566)$ & 0.141 \\
\hline CTCs before chemo & $2.847(1.613-5.024)$ & 0.0003 & $3.623(1.681-7.812)$ & 0.001 \\
\hline EFC\#93 before chemo & $3.782(1.965-7.281)$ & $<0.0001$ & $5.973(2.634-13.542)$ & $<0.0001$ \\
\hline
\end{tabular}

Cox proportional hazards models. All statistical tests were two-sided

Cl confidence interval, CTC circulating tumor cell, HR hazard ratio 

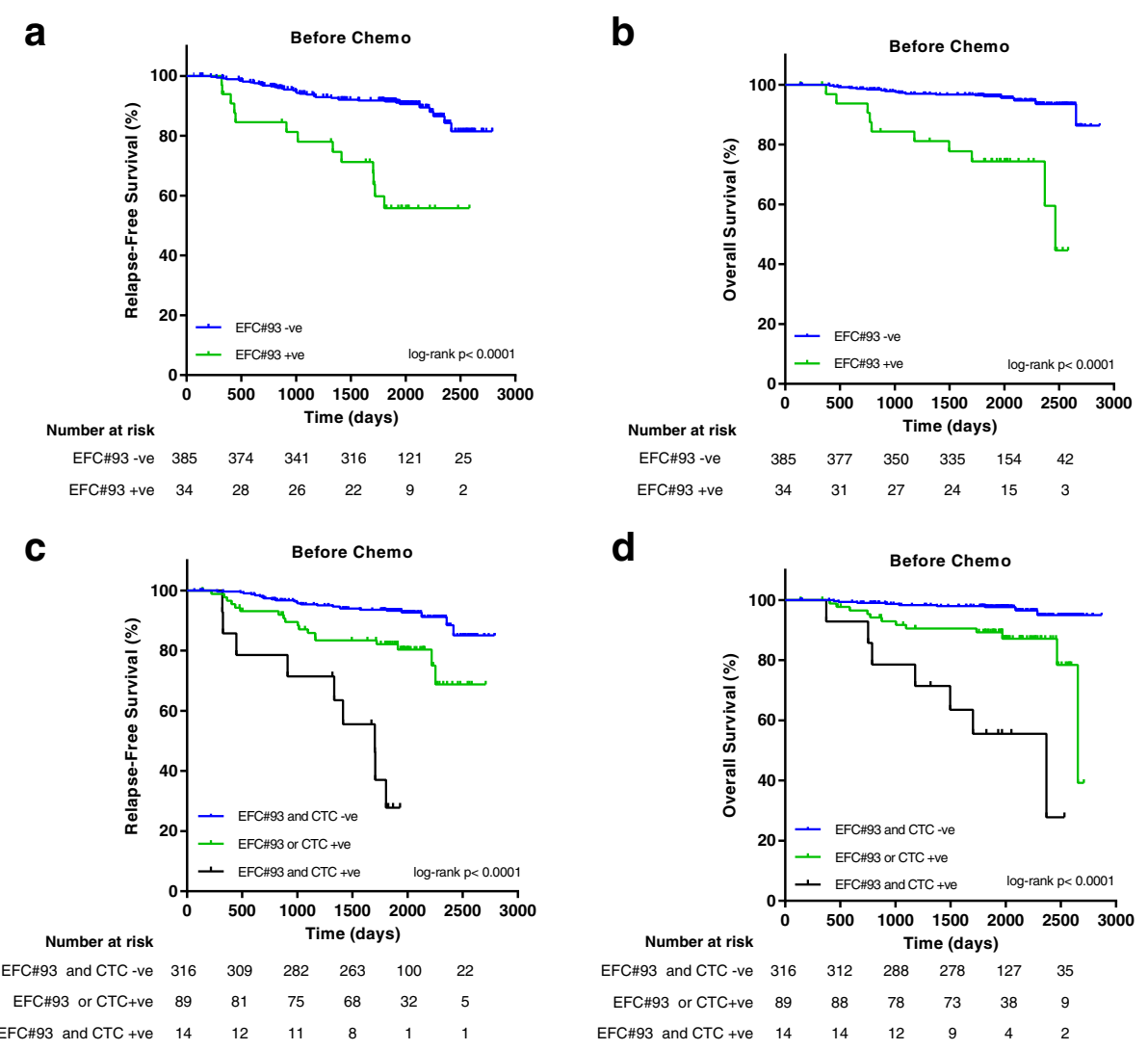

Fig. 3 EFC\#93 serum DNAme and CTC analyses in the SUCCESS trial in samples taken before chemotherapy. Kaplan-Meier analysis for relapse-free survival (a) and overall survival (b) according to the presence (EFC\#93 pattern frequency $\geq 0.00008$ ) or absence (EFC\#93 pattern frequency <0.00008) of marker EFC\#93 before chemotherapy. Kaplan-Meier analysis for relapse-free survival (c) and overall survival (d) according to the presence/absence of EFC\#93 and CTCs. $P$ values from a two-sided log-rank test. CTC- no CTC present, CTC+ at least one CTC present

from lysed blood cells would impact on assay sensitivity, particularly in a pre-clinical setting where only traces of cancer DNA were expected in the circulation. We therefore split all samples into two groups: (1) low serum DNA amount; and (2) high serum DNA amount. In the "low DNA" group, we observed a significantly higher EFC\#93 serum DNAme pattern frequency in the women who developed $\mathrm{BC}$ within one year after sample donation and subsequently died (Fig. 4a; cut-off threshold of 0.00008). Due to the high levels of background DNA, no significant findings were observed in the "high DNA" sample groups (Fig. 4b). In the "low DNA" group, EFC\#93 DNAme was able to identify $43 \%$ of women 3-6 months and $25 \%$ of women $6-12$ months before the diagnosis of a BC which eventually led to death, with a specificity of $88 \%$ (Fig. 4c). The sensitivity of serum EFC\#93 methylation in detecting fatal $\mathrm{BCs}$ up to one year in advance of diagnosis was $\sim 4$-fold higher compared to non-fatal BCs $(33.9 \%$ compared to $9.3 \%)$. In fact, the sensitivity for non-fatal $\mathrm{BCs}$ was within the false-positive range of the healthy samples, indicating that non-fatal BCs are not detected with this marker.

\section{Discussion}

We demonstrate that our serum DNAme marker, EFC\#93, can be detected up to one year in advance of BC diagnosis and is a marker for poor prognosis in the adjuvant primary treatment setting. EFC\#93 is located within GP5, a gene coding for a surface glycoprotein which has been suggested to be involved in hematogenous breast cancer metastasis [36].

The use of tumor-specific methylated DNA in serum using targeted ultra-high bisulfite sequencing has the following advantages compared to alternative strategies: (1) patient plasma/serum DNA can be amplified to increase assay sensitivity; (2) abnormal DNAme is a stable tumorspecific marker occurring early in carcinogenesis and is conserved throughout disease progression [22]; (3) selection of $\mathrm{CpG}$ island hypermethylation simplifies assay design; and (4) DNAme over several linked CpGs 

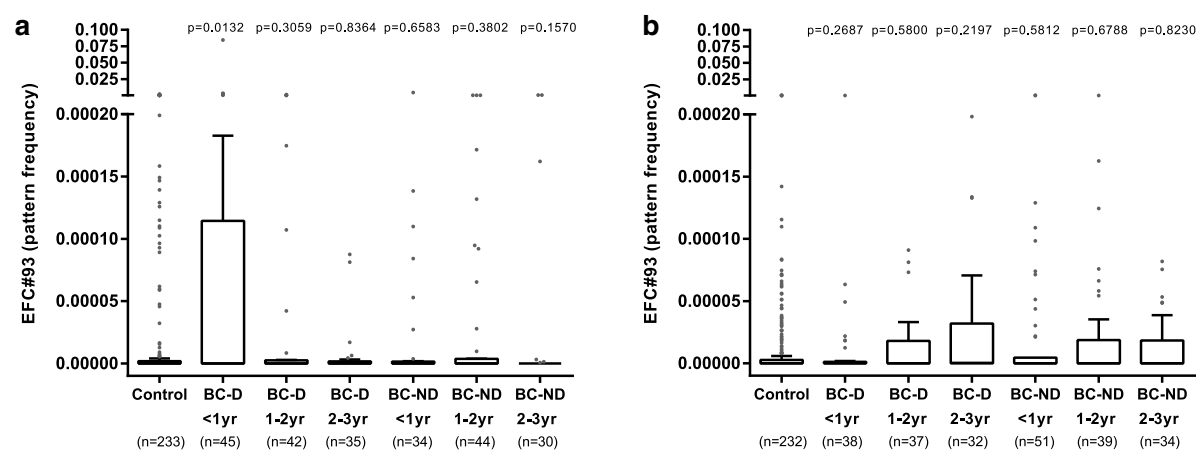

C

\begin{tabular}{|cccc|}
\hline & & Low DNA amount & High DNA amount \\
\hline & Specificity & $88.0 \%(82.9-91.7)$ & $96.1 \%(92.5-98.1)$ \\
\hline \multirow{3}{*}{$\begin{array}{c}\text { Breast Cancer } \\
\text { DECEASED }\end{array}$} & Sensitivity 3-6mo & $42.9 \%(18.8-70.4)$ & $0 \%(0.0-24.1)$ \\
& Sensitivity 6-12mo & $25.0 \%(11.4-88.6)$ & $0 \%(0.0-25.3)$ \\
& Sensitivity 1-2yr & $16.7 \%(7.5-32.0)$ & $5.4 \%(0.9-19.5)$ \\
& Sensitivity 2-3yr & $5.7 \%(1.0-20.5)$ & $5.4 \%(0.9-19.5)$ \\
\hline \multirow{2}{*}{$\begin{array}{c}\text { Breast Cancer } \\
\text { ALIVE }\end{array}$} & Sensitivity 3-6mo & $9.1 \%(0.5-42.9)$ & $10.5 \%(1.8-34.5)$ \\
& Sensitivity 6-12mo & $9.5 \%(1.7-31.8)$ & $12.0 \%(3.2-32.3)$ \\
& Sensitivity 1-2yr & $15.4 \%(6.4-31.2)$ & $5.7 \%(1.0-20.5)$ \\
& Sensitivity 2-3yr & $11.4 \%(3.7-27.7)$ & $5.3 \%(0.9-19.1)$ \\
\hline
\end{tabular}

Fig. 4 Pattern frequency of EFC\#93 in women from the UKCTOCS. EFC\#93 pattern frequency in samples with low (a) or high (b) amounts of DNA in the serum sample. $\mathbf{c}$ Performance of EFC\#93 serum DNAme marker (cut-off threshold $=0.00008$ ) depending on time to diagnosis and whether or not women subsequently died. Data separated based on DNA amount in the serum sample ( $95 \% \mathrm{Cl}$ in brackets). $P$ values in (a) and (b) are from a Mann-Whitney U-test and are relative to the control group. Control no cancer developed, BC-D breast cancer which eventually led to death, BC-ND breast cancer which did not lead to death, mo months, yr years

constitutes a clearly detectable signal with a higher specificity (due to alleviated sensitivity to sequencing errors).

A key limitation of any current large-scale populationbased cell-free DNA study, such as ours, is the lack of highquality samples. This was evident in both the SUCCESS and UKCTOCS samples, where the blood samples were not processed until 24-96 h after the blood was drawn and hence contained large amounts of leaked WBC DNA. In healthy individuals, cell-free DNA is normally present at concentrations in the range of $0-100 \mathrm{ng} / \mathrm{mL}$ and at an average of $30 \mathrm{ng} / \mathrm{mL}$ [37]. DNA derived from tumor cells is also shorter than that from non-malignant cells in the plasma of cancer patients and typically $166 \mathrm{bp}$ long [38]. Blood tubes which stabilize cell-free DNA and prevent leakage of WBC DNA are now available [39] and will be used for any future studies.

The leaked DNA in these serum samples will no doubt have led to a preferential amplification of non-cancer DNA. Despite these complicating factors, EFC\#93 serum DNAme, before treatment, was a strong prognostic factor and was complementary to CTCs. Some previous studies on CTCs used a cut-off value of $>5$ cells $/ \mathrm{mL}$; this may certainly be valid and useful for metastatic BC patients. In the SUCCESS setting of primary $\mathrm{BC}$ patients, only $8 / 419$ patients $(1.9 \%)$ had $>5$ CTCs $/ \mathrm{mL}$. Had we taken this CTC cut-off, the relapse-free survival HR would have been 4.8 with a relatively wide $95 \%$ CI of $1.5-15.5(P=0.009)$. Hence, the chosen threshold that we pre-specified in previous work [10] (i.e. CTCs detectable or not) is completely justified in this primary cancer setting.

For the current genetic cell-free DNA markers the detection limit is in the range of $0.1 \%$ allele frequency (i.e. $1 \mathrm{mu}$ tated in the background of 1000 non-mutated alleles can be detected $[15,21])$. Ultra-high coverage bisulfite-sequencing, however, allows for far more sensitive testing. Mammography screening in women aged $50-75$ years has a sensitivity of $82-86 \%$ and a specificity of $88-92 \%$ for detecting any $\mathrm{BC}$; however, the majority of these cancers are not fatal [40]. EFC\#93 serum DNAme has a sensitivity of $43 \%$ in identifying fatal breast cancer up to six months in advance of current diagnosis at a similar specificity $(88 \%)$ to mammography, supporting the rationale for incorporating serum DNAme markers in future cancer-screening trials.

Based on the evidence accumulated so far, we have to assume that EFC\#93 indicates the presence of disseminated breast cancer, which at least in a proportion of women, will not yet be clinically evident in the breast. Hence, the question arises whether EFC\#93-positive 
mammography-negative women should watch and wait (i.e. within an enhanced surveillance program) or whether this group of women could also be offered a strategy which actively deals with the likely disseminated disease until radiological evidence in the breast starts to arise. Anti-hormonal treatment (i.e. Tamoxifen or aromatase inhibitors) are being used for both adjuvant and preventive treatment. Therefore, we assessed whether EFC\#93 positivity after SUCCESS chemotherapy (which is before the initiation of anti-hormonal treatment) is associated with survival: EFC\#93 positivity in post-chemotherapy samples of hormone receptor-negative women still indicated a poor prognosis whereas EFC\#93 positivity in hormone receptor-positive women was no longer associated with poor prognosis (Additional file 1: Figure S14). CTC status in post-chemotherapy samples was not associated with outcome irrespective of subsequent anti-hormonal treatment (Additional file 1: Figure S15).

\section{Conclusions}

Overall and for the first time, our study provides evidence that serum DNAme markers can diagnose fatal $\mathrm{BCs}$ up to one year in advance of current diagnosis and enable individualized $\mathrm{BC}$ treatment which may even commence before obtaining radiological evidence in the breast. In addition, the combination of CTC and cellfree DNA analysis might further improve risk stratification of breast cancer patients. The recent advance of purposed blood tubes will facilitate clinical implementation of DNAme pattern detection of cell-free DNA as a clinical tool in cancer medicine.

\section{Additional files}

Additional file 1: Figure S1. Samples from the SUCCESS trial analyzed within this study. Figure S2. Samples from the UKCTOCS cohort analyzed within this study (nested case/control setting). Figure S3. Absolute pattern counts for all patterns detected in the region of marker EFC\#93 in Serum Set 1 samples. Figure S4. Pattern frequency of EFC\#93 serum DNAme in two prospectively independently collected cohorts. Figure S5. DNA amount per $\mathrm{mL}$ serum in the prospectively collected serum (Set 1 and 2), SUCCESS cohort, and UKCTOCS cohort. Figure S6. Pattern frequency for EFC\#93 measured in SUCCESS serum set samples from women with no, 1-4 or $\geq 5$ CTCS in the matched blood sample before (A) or after (B) chemotherapy. Figure S7. Impact of the presence (+ve, $\geq 1$ cancer cell in blood sample) or absence (-ve) of CTCs on patient outcome. Figure S8. Impact of the presence (+ve, EFC\#93 pattern frequency $\geq 0.00008$ ) or absence (-ve) of serum DNA methylation in CTC + ve ( $\geq 1$ cancer cell in pre-chemotherapy blood sample) or absence CTC-ve patients. Figure S9. Relapse-free and overall survival according to samples taken after chemotherapy. Figure S10. Relapse-free and overall survival according to samples taken after chemotherapy. Figure S11. Average serum DNA amount correlates with average UK temperature. Figure S12. Average serum DNA fragment size correlates with average UK temperature. Figure S13. Correlation of DNA fragment size and DNA amount. Figure S14. Overall survival of women whose samples were taken before and after chemotherapy and before anti-hormonal treatment in hormone receptor-negative and -positive SUCCESS participants. Figure S15. Overall survival of women whose samples were taken before and after chemotherapy and before anti-hormonal treatment in hormone receptor-negative and -positive SUCCESS participants. (PDF 2123 kb)

Additional file 2: Supplementary Material and Methods: Additional details of samples sets, methods and analyses. (PDF 279 kb)

Additional file 3: Table S1. Coordinates and primers used to amplify the identified target regions using bisulfite sequencing. (PDF $578 \mathrm{~kb}$ )

\section{Abbreviations}

AUC: Area under the curve; BC: Breast cancer; bp: Base pairs; CTC: Circulating tumor cells; DNAme: DNA methylation; ROC: Receiver operating characteristics; WBC: White blood cell

\section{Acknowledgements}

We thank Marina Koßmann-Plasa, Verena Schindler, Sascha Vugrinec, Julia Bottlang, Eleni Pachygiannaki, Nadine Mall, Efrain Manilla-Pérez, Jacqueline Berner, Alexander Krämer, and Verena Mark for excellent technical assistance. Further, thanks go to Kapil Jhalani, Yadhu Kumar, and Fabian Ripp for their assistance in data analysis. We are also grateful to Markus Schmitt and Kerstin Rönsch for their support and fruitful discussions. We thank all the trial participants for their generosity in donating samples for secondary studies and all the staff involved in the SUCCESS and UKCTOCS trials.

\section{Funding}

This work was funded by the European Union's Seventh Framework Programme (FP7/2007-2013) under grant agreement number 305428 (Project EpiFemCare). MW also received support from The Eve Appeal (https:// eveappeal.org.uk/). Part of this research was undertaken at UCLH/UCL, which received a proportion of its funding from the Department of Health NIHR Biomedical Research Centres funding scheme.

UKCTOCS was funded by the Medical Research Council (G9901012 and G0801228), Cancer Research UK (C1479/A2884), and the Department of Health, with additional support from The Eve Appeal.

\section{Availability of data and materials}

The RRBS data are available in the European Genome-Phenome Archive in order to allow controlled access under the accession number EGAS00001002609.

\section{Authors' contributions}

The study was conceived and designed by MW. DNA methylation analyses and other experimental work were undertaken by BW, TP, IE, and AJ. Statistical analysis was performed by $\mathrm{HL}$ and TW with support from JE. UM and AGM contributed to the design of the UKCTOCS validation study. The manuscript was written by MW with support from IE, AJ, DR, SG, AR, AG-M, MZ, DC, MA-F, $J \mathrm{~K}$, WJJ, UM, and BR. All authors read and approved the manuscript.

\section{Ethics approval and consent to participate}

Samples were collected prospectively at the University College London Hospital in London and at the Charles University Hospital in Praque and the Department of Gynaecology and Obstetrics, Klinikum Innenstadt, Ludwig-Maximilians-Universitaet Muenchen, Maistr. 11, 80337 Munich, Germany. The study was approved by the local research ethics committees (UCL/UCLH Biobank for Studying Health \& Disease NC09.13 and the ethics committee of the General University Hospital, Prague approval no:: 22/13 GRANT - 7. RP - EPI-FEM-CARE) as well as by the ethical committee of the Ludwig-Maximilians-University Munich. The SUCCESS study was approved by 37 German ethical boards (lead ethical board: Ludwig-MaximiliansUniversity Munich). All patients provided written informed consent. The UKCTOCS study was approved by the local research ethics committees (UCL/UCLH Biobank for Studying Health \& Disease NC09.13) and was approved as part of trial approval by the UK North West Multicentre Research Ethics Committees (North West MREC 00/8/34). All patients provided written informed consent and all studies were conducted in accordance with the Declaration of Helsinki.

\section{Consent for publication}

Not applicable.

\section{Competing interests}

$\mathrm{HL}$ is an employee and shareholder of Genedata AG. UM has stock ownership in and research funding from Abcodia Pvt Ltd which has an 
interest in cancer biomarkers. The remaining authors declare that they have no competing interests.

\section{Publisher's Note}

Springer Nature remains neutral with regard to jurisdictional claims in published maps and institutional affiliations.

\section{Author details}

'Department of Women's Cancer, UCL Elizabeth Garrett Anderson Institute for Women's Health, University College London, Medical School Building, 74 Huntley Street, London WC1E 6AU, UK. ${ }^{2}$ Gynaecologic Oncology Center, Department of Obstetrics \& Gynaecology, First Faculty of Medicine \& General University Hospital, Charles University Prague, Prague, Czech Republic. ${ }^{3}$ Genedata AG, Margarethenstrasse 38, 4053 Basel, Switzerland. ${ }^{4}$ Department of Gynaecology and Obstetrics, Klinikum Innenstadt, Ludwig-Maximilians Universitaet Muenchen, Maistrasse 11, 80337 Munich, Germany. ${ }^{5}$ Department of Gynaecology and Obstetrics, University Hospital Ulm, Prittwitzstrasse 43, 89075 Ulm, Germany. ${ }^{6}$ GATC Biotech AG, Jakob-Stadler-Platz 7, 78467 Konstanz, Germany. ${ }^{7}$ Boehringer Ingelheim Pharma, GmbH \& Co. KG, Target Discovery Research, Biberach, Germany.

\section{Received: 19 May 2017 Accepted: 22 November 2017}

Published online: 22 December 2017

\section{References}

1. Torre LA, Bray F, Siegel RL, Ferlay J, Lortet-Tieulent J, Jemal A. Global cancer statistics, 2012. CA Cancer J Clin. 2015;65(2):87-108.

2. Marmot MG, Altman DG, Cameron DA, Dewar JA, Thompson SG, Wilcox M. The benefits and harms of breast cancer screening: an independent review. Br J Cancer. 2013;108(11):2205-40.

3. Harper KL, Sosa MS, Entenberg D, Hosseini H, Cheung JF, Nobre R, et al. Mechanism of early dissemination and metastasis in Her2+ mammary cancer. Nature. 2016;540:588-92.

4. Kazarian A, Blyuss O, Metodieva G, Gentry-Maharaj A, Ryan A, Kiseleva EM, et al. Testing breast cancer serum biomarkers for early detection and prognosis in pre-diagnosis samples. $\mathrm{Br} \mathrm{J}$ Cancer. 2017;116(4):501-8

5. Braun S, Vogl FD, Naume B, Janni W, Osborne MP, Coombes RC, et al. A pooled analysis of bone marrow micrometastasis in breast cancer. $\mathrm{N}$ Engl J Med. 2005:353(8):793-802.

6. Mansi JL, Gogas H, Bliss JM, Gazet JC, Berger U, Coombes RC. Outcome of primary-breast-cancer patients with micrometastases: a long-term follow-up study. Lancet. 1999:354(9174):197-202.

7. Klein CA, Blankenstein TJ, Schmidt-Kittler O, Petronio M, Polzer B, Stoecklein $\mathrm{NH}$, et al. Genetic heterogeneity of single disseminated tumour cells in minimal residual cancer. Lancet. 2002;360(9334):683-9.

8. Bidard FC, Peeters DJ, Fehm T, Nole F, Gisbert-Criado R, Mavroudis D, et al. Clinical validity of circulating tumour cells in patients with metastatic breast cancer: a pooled analysis of individual patient data. Lancet Oncol. 2014; 15(4):406-14.

9. Lucci A, Hall CS, Lodhi AK, Bhattacharyya A, Anderson AE, Xiao L, et al. Circulating tumour cells in non-metastatic breast cancer: a prospective study. Lancet Oncol. 2012;13(7):688-95.

10. Rack B, Schindlbeck C, Juckstock J, Andergassen U, Hepp P, Zwingers T, et al. Circulating tumor cells predict survival in early average-to-high risk breast cancer patients. J Natl Cancer Inst. 2014;106(5):dju066.

11. Cristofanilli M, Budd GT, Ellis MJ, Stopeck A, Matera J, Miller MC, et al. Circulating tumor cells, disease progression, and survival in metastatic breast cancer. N Engl J Med. 2004;351(8):781-91.

12. Janni WJ, Rack B, Terstappen LW, Pierga JY, Taran FA, Fehm T, et al. Pooled analysis of the prognostic relevance of circulating tumor cells in primary breast cancer. Clin Cancer Res. 2016;22(10):2583-93.

13. Welch HG, Prorok PC, O'Malley AJ, Kramer BS. Breast-cancer tumor size, overdiagnosis, and mammography screening effectiveness. N Engl J Med. 2016:375(15):1438-47.

14. Klein CA. Parallel progression of primary tumours and metastases. Nat Rev Cancer. 2009:9(4):302-12

15. Dawson SJ, Tsui DW, Murtaza M, Biggs H, Rueda OM, Chin SF, et al. Analysis of circulating tumor DNA to monitor metastatic breast cancer. N Engl J Med. 2013;368(13):1199-209.
16. Murtaza M, Dawson SJ, Tsui DW, Gale D, Forshew T, Piskorz AM, et al. Noninvasive analysis of acquired resistance to cancer therapy by sequencing of plasma DNA. Nature. 2013;497(7447):108-12.

17. Wang Y, Springer S, Mulvey CL, Silliman N, Schaefer J, Sausen M, et al. Detection of somatic mutations and HPV in the saliva and plasma of patients with head and neck squamous cell carcinomas. Sci Transl Med. 2015;7(293):293ra104.

18. Siravegna G, Mussolin B, Buscarino M, Corti G, Cassingena A, Crisafulli G, et al. Clonal evolution and resistance to EGFR blockade in the blood of colorectal cancer patients. Nat Med. 2015;21(7):827.

19. Bettegowda C, Sausen M, Leary RJ, Kinde I, Wang Y, Agrawal N, et al. Detection of circulating tumor DNA in early- and late-stage human malignancies. Sci Transl Med. 2014;6(224):224ra224.

20. De Mattos-Arruda L, Caldas C. Cell-free circulating tumour DNA as a liquid biopsy in breast cancer. Mol Oncol. 2016;10(3):464-74.

21. Lanman RB, Mortimer SA, Zill OA, Sebisanovic D, Lopez R, Blau S, et al. Analytical and clinical validation of a digital sequencing panel for quantitative, highly accurate evaluation of cell-free circulating tumor DNA. PLoS One. 2015:10(10):e0140712

22. Baylin SB, Jones PA. A decade of exploring the cancer epigenome - biological and translational implications. Nat Rev Cancer. 2011:11(10):726-34.

23. Teschendorff AE, Gao Y, Jones A, Ruebner M, Beckmann MW, Wachter DL, et al. DNA methylation outliers in normal breast tissue identify field defects that are enriched in cancer. Nat Commun. 2016;7:10478.

24. Fackler MJ, Lopez BZ, Umbricht C, Teo WW, Cho S, Zhang Z, et al. Novel methylated biomarkers and a robust assay to detect circulating tumor DNA in metastatic breast cancer. Cancer Res. 2014;74(8):2160-70.

25. Fiegl H, Millinger S, Mueller-Holzner E, Marth C, Ensinger C, Berger A, et al. Circulating tumor-specific DNA: a marker for monitoring efficacy of adjuvant therapy in cancer patients. Cancer Res. 2005;65(4):1141-5.

26. Muller HM, Widschwendter A, Fiegl H, Ivarsson L, Goebel G, Perkmann E, et al. DNA methylation in serum of breast cancer patients: an independent prognostic marker. Cancer Res. 2003;63(22):7641-5.

27. Muller HM, Fiegl H, Widschwendter A, Widschwendter M. Prognostic DNA methylation marker in serum of cancer patients. Ann N Y Acad Sci. 2004; 1022:44-9.

28. Warton K, Mahon KL, Samimi G. Methylated circulating tumor DNA in blood: power in cancer prognosis and response. Endocr Relat Cancer. 2016; 23(3):R157-71.

29. Wittenberger T, Sleigh S, Reisel D, Zikan M, Wahl B, Alunni-Fabbroni M, et al. DNA methylation markers for early detection of women's cancer: promise and challenges. Epigenomics. 2014;6(3):311-27.

30. Sun K, Jiang P, Chan KC, Wong J, Cheng YK, Liang RH, et al. Plasma DNA tissue mapping by genome-wide methylation sequencing for noninvasive prenatal, cancer, and transplantation assessments. Proc Natl Acad Sci U S A. 2015;112(40):E5503-12

31. Visvanathan K, Fackler MS, Zhang Z, Lopez-Bujanda ZA, Jeter SC, Sokoll LJ, et al. Monitoring of serum DNA methylation as an early independent marker of response and survival in metastatic breast cancer: TBCRC 005 Prospective Biomarker Study. J Clin Oncol. 2017;35(7):751-8.

32. Jacobs IJ, Menon U, Ryan A, Gentry-Maharaj A, Burnell M, Kalsi JK, et al. Ovarian cancer screening and mortality in the UK Collaborative Trial of Ovarian Cancer Screening (UKCTOCS): a randomised controlled trial. Lancet. 2016;387(10022):945-56

33. Gu H, Smith ZD, Bock C, Boyle P, Gnirke A, Meissner A. Preparation of reduced representation bisulfite sequencing libraries for genome-scale DNA methylation profiling. Nat Protoc. 2011;6(4):468-81.

34. Lee YK, Jin S, Duan S, Lim YC, Ng DP, Lin XM, et al. Improved reduced representation bisulfite sequencing for epigenomic profiling of clinical samples. Biol Proced Online. 2014;16(1):1.

35. Newcombe RG. Two-sided confidence intervals for the single proportion: comparison of seven methods. Stat Med. 1998;17(8):857-72.

36. Suter CM, Hogg PJ, Price JT, Chong BH, Ward RL. Identification and characterisation of a platelet GPIb/N/IX-like complex on human breast cancers: implications for the metastatic process. Jpn J Cancer Res. 2001; 92(10):1082-92.

37. Gormally E, Caboux E, Vineis $P$, Hainaut $P$. Circulating free DNA in plasma or serum as biomarker of carcinogenesis: practical aspects and biological significance. Mutat Res. 2007;635(2-3):105-17.

38. Jiang P, Lo YM. The long and short of circulating cell-free DNA and the ins and outs of molecular diagnostics. Trends Genet. 2016;32(6):360-71. 
39. Kang $Q$, Henry NL, Paoletti C, Jiang H, Vats P, Chinnaiyan AM, et al. Comparative analysis of circulating tumor DNA stability in KEDTA, Streck, and Cell Save blood collection tubes. Clin Biochem. 2016;49:1354-60.

40. Fenton JJ, Taplin SH, Carney PA, Abraham L, Sickles EA, D'Orsi C, et al. Influence of computer-aided detection on performance of screening mammography. N Engl J Med. 2007;356(14):1399-409.

Submit your next manuscript to BioMed Central and we will help you at every step:

- We accept pre-submission inquiries

- Our selector tool helps you to find the most relevant journal

- We provide round the clock customer support

- Convenient online submission

- Thorough peer review

- Inclusion in PubMed and all major indexing services

- Maximum visibility for your research

Submit your manuscript at www.biomedcentral.com/submit 